\title{
Health care in the United States: A corporate industry, no longer a professional enterprise
}

\author{
David H Van Thiel* \\ Director of Advanced liver and Gastrointestinal Disease Center, Berwyn, 1llinois,60402, USA
}

Health care in the United States has experienced a progressive loss of professionalism beginning with the decision to accept direct third-party reimbursement for professional services rendered to their patients. The derivative of this fatal decision was an increasing relationship between hospitals and insurers. This led directly to the aggressive recruitment of large numbers of primary care physicians by hospitals followed sequentially by the recruitment of a smaller number of hospital-based subspecialists.

This changed the ethics of medical care wherein primary care physicians were required to refer solely to their corporate hospitalbased subspecialists regardless of the experience and/or skill of the corporate subspecialist. They were prohibited from referring to a more skilled subspecialist outside the hospital corporate structure. With the continued expansion of the hospital based corporate enterprise, managed by businessman (MBAs, Accountants etc.) rather than physicians, the emphasis on medical care shifted from individual physician based quality care provided to the patient (client) to corporate medical care meeting "the standard of care" (least common denominator) utilizing a conveyer belt-like approach wherein the number of units (patient encounters) rather than the quality of care was important. Little or no attention was given to the concepts of the Hippocratic Oath as the primary goal. This new approach shifting healthcare delivery from that practiced by a professional physician to a corporate physician employee has de facto eliminated professionalism in the practice of medicine.

Moreover, reimbursement was determined by "units" of service rather than the quality, amount of professional time expended, or complexity of the services provided contributed to the loss of professionalism in the practice of medicine. Professionals, whether they are barbers, hairdressers, mechanics, or lawyers, receive increasing pay for service as a function of their skill and experience not units of service. Once the sense of professionalism is lost and the individual's status as an employee is accepted, the services provided the individual client (patient) is determined by the corporate employer rather than the physician.

This corporate management concept has expanded beyond physicians to pharmacies wherein contractual agreements between

Copyright: (C2018 Van Thiel DH. This is an open-access article distributed under the terms of the Creative Commons Attribution License, which permits unrestricted use, distribution, and reproduction in any medium, provided the original author and source are credited. health care insurers and corporate pharmacies determine the specific therapeutic agents can be utilized rather than what the health care provider (physician) thinks would be best for the client (patient). The choice of any given therapeutic agent is determined by the corporate formulary to maximize net reimbursement with the elimination of more expensive potentially more effective agents. Recently, a corporate pharmacy (CVS) has acquired a health care insurer (Aetna) creating a single larger corporate structure which raises conflict of interest (anti-trust) concerns. In addition, ndividual freestanding hospitals are progressively being eliminated by large corporate networks of hospitals in an effort to maximize income and are no longer "hospitals" but rather a combination of an emergency room combined with an extended emergent care unit and a surgical facility. "Hospital care" as previously defined is currently delivered solely on an outpatient basis.

All of these changes in health care provision have eliminated the concept of collegiality between physicians, hospitals and their clients (patients). As a result of these changes in health care, doctors are no longer professionals but have become "corporate providers". This has led to the concept that all physicians are identical in terms of their skill and knowledge and provide the same "standard of care medicine" for the corporation. Even more troublesome is the reality that physicians or no longer allowed to identify disease processes early and prevent later complications but rather can respond only to symptoms or signs of overt disease. An example of this reality is that insurers will only reimburse an ultrasound examination of the carotid arteries if the individual has a stroke or bruit notwithstanding the presence of extensive cardiovascular disease suggesting that the underlying cardiovascular disease extends to the carotid arteries as well. Another example of this approach is that for all practical purposes, prevention in the current corporate approach to medicine is not a reality. Vaccinations for example are rarely reimbursed by health care insurers.

In summary, the current corporate approach to healthcare delivery enhances the cost of care, corporate profitability, and deprofessionalizes physicians, but whether it improves the health of the population at large remains to be shown. 